\title{
TaALMT1 promoter sequence compositions, acid tolerance, and Al tolerance in wheat cultivars and landraces from Sichuan in China
}

C. Han ${ }^{1 *}$, S.F. Dai ${ }^{1 *}$, D.C. Liu ${ }^{1}$, Z.J. Pu' ${ }^{2}$, Y.M. Wei', Y.L. Zheng', D.J. Wen', L. Zhao ${ }^{1}$ and Z.H. Yan $^{1}$

${ }^{1}$ Triticeae Research Institute, Sichuan Agricultural University, Chengdu, Sichuan, China

${ }^{2}$ Crop Research Institute, Sichuan Academy of Agricultural Science, Chengdu, Sichuan, China

*These authors contributed equally to this study.

Corresponding author: Z.-H. Yan

E-mail: zhyan104@163.com

Genet. Mol. Res. 12 (4): 5602-5616 (2013)

Received November 8, 2012

Accepted February 4, 2013

Published November 18, 2013

DOI http://dx.doi.org/10.4238/2013.November.18.9

\begin{abstract}
Previous genetic studies on wheat from various sources have indicated that aluminum (Al) tolerance may have originated independently in USA, Brazil, and China. Here, TaALMT1 promoter sequences of 92 landraces and cultivars from Sichuan, China, were sequenced. Five promoter types (I', II, III, IV, and V) were observed in 39 cultivars, and only three promoter types (I, II, and III) were observed in 53 landraces. Among the wheat collections worldwide, only the Chinese Spring (CS) landrace native to Sichuan, China, carried the TaALMT1 promoter type III. Besides CS, two other Sichuan-bred landraces and six cultivars with TaALMT1 promoter type III were identified in this study. In the phylogenetic tree constructed based on the TaALMT1 promoter sequences, type III formed a separate branch, which was supported by a high bootstrap value. It is likely that TaALMT1 promoter type III originated from Sichuan-bred wheat landraces of China. In addition, the
\end{abstract}


landraces with promoter type I showed the lowest Al tolerance among all landraces and cultivars. Furthermore, the cultivars with promoter type IV showed better Al tolerance than landraces with promoter type II. A comparison of acid tolerance and Al tolerance between cultivars and landraces showed that the landraces had better acid tolerance than the cultivars, whereas the cultivars showed better Al tolerance than the landraces. Moreover, significant difference in Al tolerance was also observed between the cultivars raised by the National Ministry of Agriculture and by Sichuan Province. Among the landraces from different regions, those from the East showed better acid tolerance and $\mathrm{Al}$ tolerance than those from the South and West of Sichuan. Additional Al-tolerant and acid-tolerant wheat lines were also identified.

Key words: Sichuan-bred wheat cultivars and landraces; Aluminum tolerance; Acid tolerance; TaALMT1 promoter

\section{INTRODUCTION}

Acid soils occupy $\sim 30 \%$ of the total ice-free land area (von Uexküll and Mutert, 1995). In soils with mildly acidic or neutral $\mathrm{pH}$ values, aluminum (Al) is primarily in an insoluble form. Below $\mathrm{pH} 5.0$, the $\mathrm{Al}$ compound often solubilizes to form toxic trivalent cations $\left(\mathrm{Al}^{3+}\right)$ that limit root growth, water uptake, and absorption of nutrient elements (Kochian, 1995). Farmers are recommended to use alkaline materials such as lime to mitigate Al toxicity in acidic soils, which is not feasible (Mclay et al., 1994). The breeding and cultivation of Altolerant crops is an alternative way to address this issue.

Although some Al-tolerant wheat lines have been identified, additional Al-tolerant germplasms are required to improve modern wheat crops (Berzonsky, 1992; Riede and Anderson, 1996; De Sousa, 1998; Hu et al., 2008). One of the most important Al-tolerant genetic sources was traced to Brazil, where acidic soils are predominant in wheat cultivation zones. Examples include the Al-tolerant genes in wheat lines BH 1146 (Ponta Grossa 1//Fronteira/ Mentana) and Atlas 66 (Frondoso//Redhart 3/Noll 28), which share the common ancestor Polyssu that originated from Brazil (Ponta Grossa 1 was selected from Polyssu, and Polyssu was a parent of Frondoso) (Berzonsky, 1992; Riede and Anderson, 1996; De Sousa, 1998; Garvin and Carver, 2003). Besides these, the Al-tolerant genes in some wheat lines such as ET8 and Carazinho were also traced to wheat from Brazil (Zhou et al., 2007).

Using several segregation populations, the major Al-tolerant locus was mapped on 4DL (Luo and Dvorák, 1996; Riede and Anderson, 1996; Milla and Gustafson, 2001; Raman et al., 2005), and Sasaki et al. (2004) cloned the TaALMT1 gene, mapping it to the same locus on chromosome 4DL (Ma et al., 2005; Raman et al., 2005). Genetic studies have shown that Al tolerance is conferred by the TaALMT1 gene encoding the TaALMT1 protein (Raman et al., 2005), which is a membrane protein that regulates Al-activated malate efflux from the rhizosphere to chelate toxic $\mathrm{Al}^{3+}$. The TaALMT1 protein function has been verified by heterologous expression in Xenopus ooctyes, rice, cultured tobacco cells, and intact barley plants (Delhaize et al., 2004; Sasaki et al., 2004; Yamaguchi et al., 2005). In addition, Al tolerance correlates to the level of TaALMT1 expression rather than to allelic differences. 
Two TaALMT1 alleles have been observed in the near-isogenic lines ET8 (Al-tolerant line TaALMT1-1) and ES8 (Al-sensitive line TaALMT1-2), and they differ in six bases that result in two amino acid changes in the TaALMT1 proteins (Sasaki et al., 2004, Raman et al., 2005). Whereas the 1-kb region downstream of TaALMT1 is well conserved and is not linked to $\mathrm{Al}$ tolerance, the $1-\mathrm{kb}$ region upstream of the TaALMT1 coding region is more variable, where at least seven different promoter types have been identified. Single nucleotide polymorphisms and variations in the tandem repeat numbers in this region are responsible, at least in part, for the different expression levels of TaALMT1 in a wide range of wheat cultivars (Sasaki et al., 2006).

Wheat landraces have adapted to local conditions for a long time, and outside their locality, they exhibit poor overall performance. Therefore, landraces are not amenable to improving yield-related traits of modern wheat, but are still valuable for improving the stressrelated traits of modern wheat. China has several wheat landraces, including Tibet wild weed wheat, Yunnan hulled wheat, Xinjiang rice wheat, and Sichuan white wheat complex (Shao et al., 1980; Dong et al., 1981; Chen et al., 1985; Yen et al., 1988). Importantly, these endemic landraces are distributed mainly in the acidic soils of South China. Therefore, natural selection for adaptation to acidic soils may produce diverse $\mathrm{Al}$ tolerance in these wheat germplasms.

Chinese Spring (CS), which has an intermediate level of TaALMT1 expression and $\mathrm{Al}$ tolerance, is a landrace native to Sichuan, China, with a unique promoter type III (Yen et al., 1988; Luo and Dvorák, 1996; Sasaki et al., 2006). Studies on the Al tolerance of Sichuan wheat is lacking. Therefore, we studied the wheat cultivars and landraces from Sichuan, China with the following objectives: 1) to evaluate the acid tolerance and Al tolerance levels in Sichuanbred wheat cultivars and landraces; 2) to investigate the differences in TaALMT1 promoter sequences between Sichuan-bred wheat cultivars and landraces; and 3) to understand the correlation between the acid and Al tolerance indices with TaALMT1 promoter sequence types.

\section{MATERIAL AND METHODS}

\section{Plant material}

A total of 92 Sichuan-bred wheat lines, including 39 cultivars and 53 landraces, were used in this study. The cultivars were developed by the Wheat Breeding Institute at Sichuan Province and the landraces were native to Sichuan, China (Table 1). Chinese Spring, a wheat landrace endemic to Sichuan, with moderate tolerance to Al, was used as the reference (Luo and Dvorák, 1996).

\section{Assessment of acid tolerance and Al tolerance in nutrient solution}

The acid tolerance and $\mathrm{Al}$ tolerance in wheat were assessed as previously described (Dai et al., 2009). Briefly, 50 seeds were surface-sterilized with sodium hypochlorite and rinsed with tap water. Seeds were germinated at room temperature for 2 days after a $12 \mathrm{~h}$ incubation at $4^{\circ} \mathrm{C}$. Fifteen seedlings with similar root lengths were selected and divided into three groups of five each for further treatment. The seedlings were floated with a plastic mesh in 7.5 $\mathrm{L}$ base hydroponic solution containing $0.4 \mathrm{mM} \mathrm{CaCl}_{2}, 0.65 \mathrm{mM} \mathrm{KNO}_{3}, 0.25 \mathrm{mM} \mathrm{MgCl}_{2}, 0.01$ $\mathrm{mM}\left(\mathrm{NH}_{4}\right)_{2} \mathrm{SO}_{4}$, and $0.04 \mathrm{mM} \mathrm{NH}_{4} \mathrm{NO}_{3}$. The $\mathrm{Al}$ stress treatments were performed at $\mathrm{pH} 4.5$, 7.0 , and 4.5 with $0.05 \mathrm{mM} \mathrm{Al}^{3+}$, with three replications. 


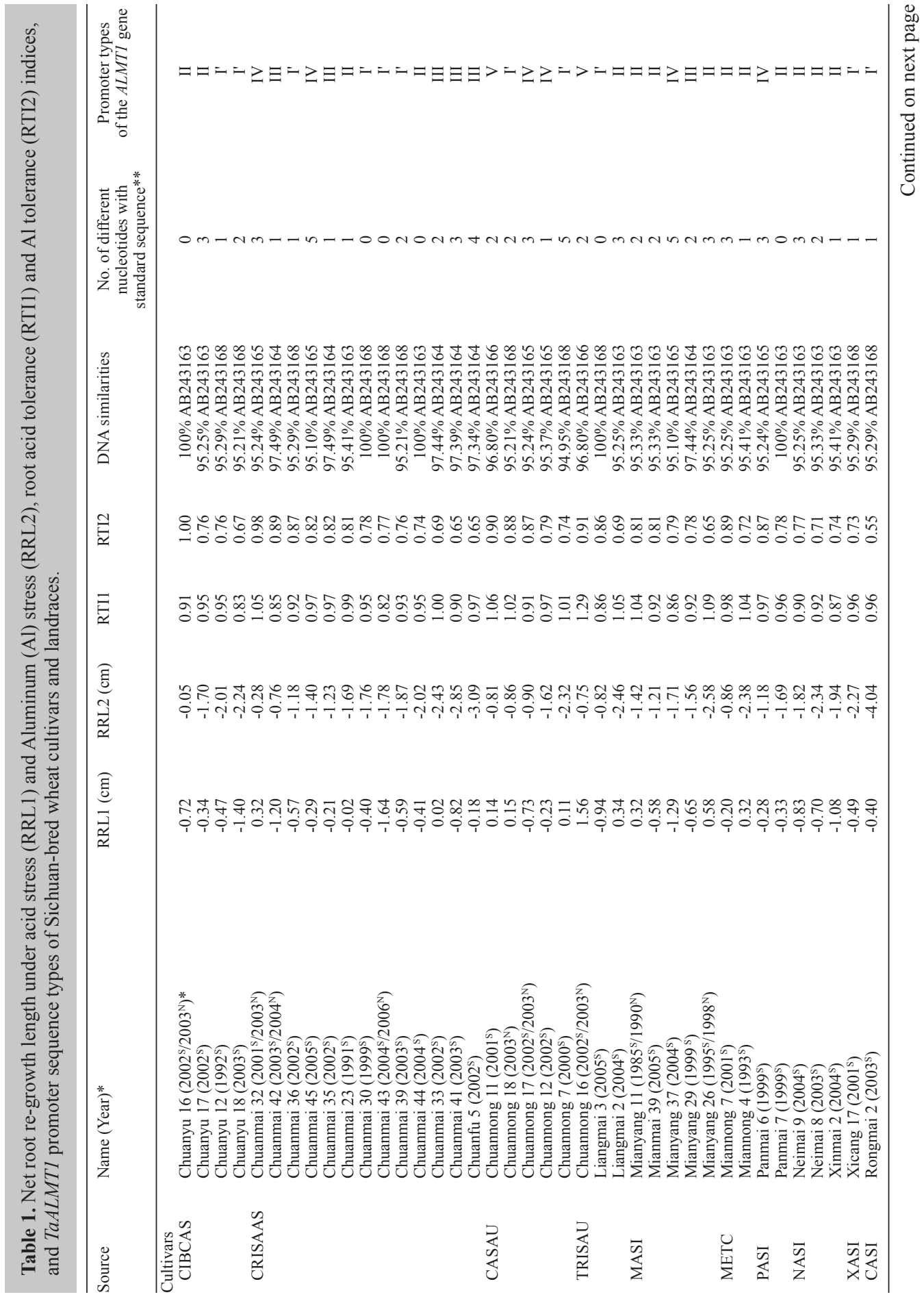




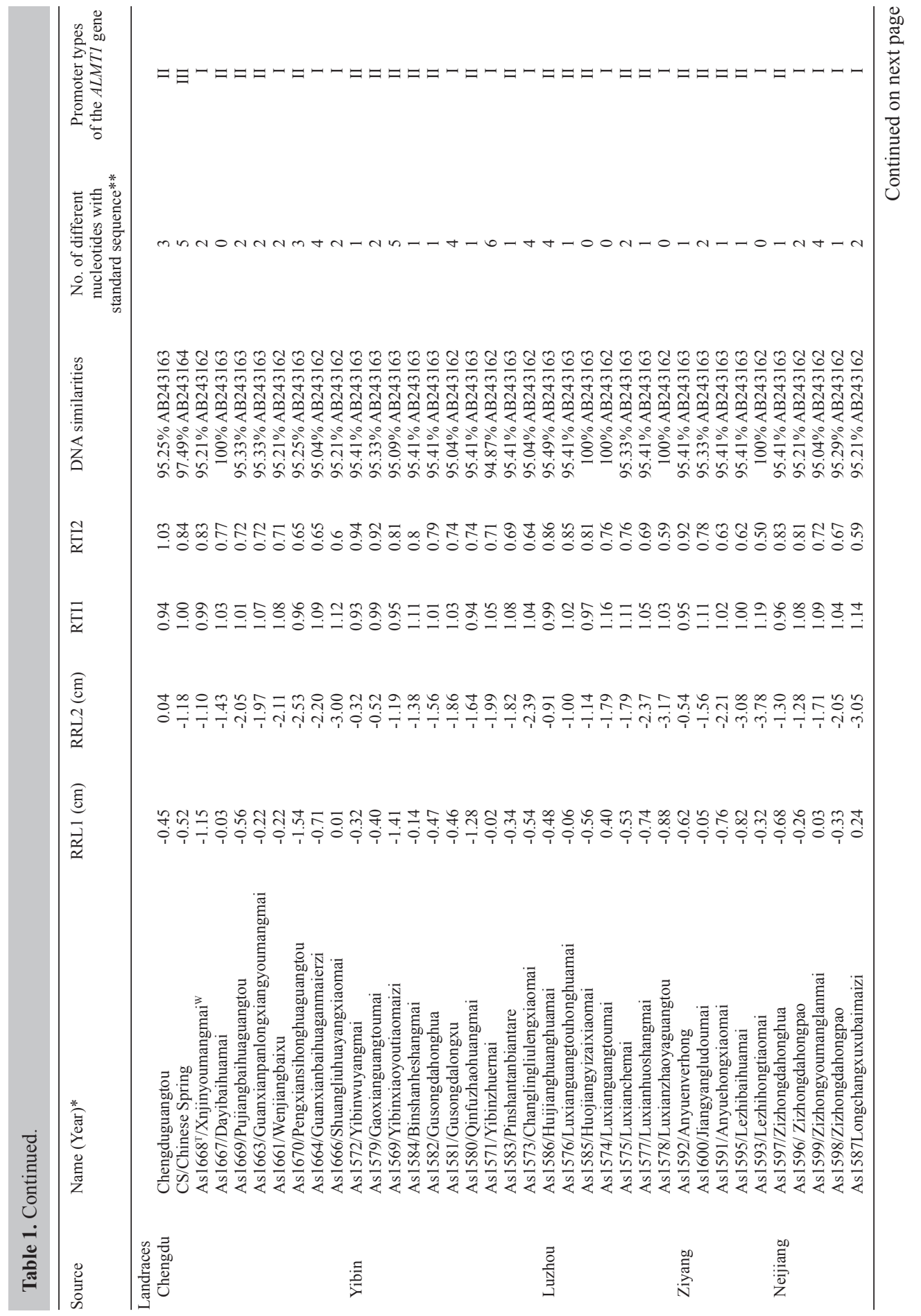


Aluminum tolerance of Sichuan-bred wheat

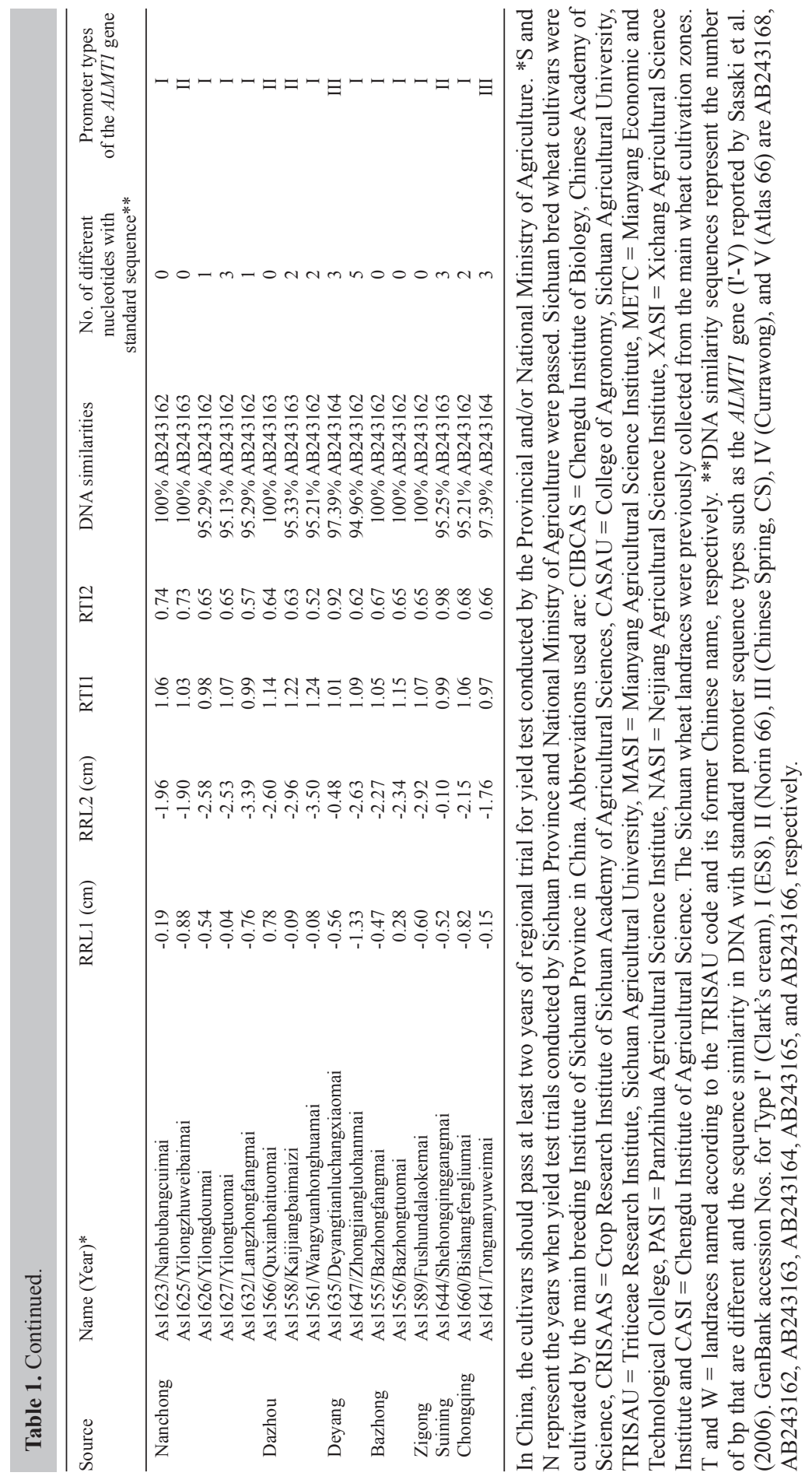


After 3 days of treatment, the root re-growth lengths (RRL) during acid stress (RRL1, $\left.=\mathrm{RRL}_{\mathrm{pH} 4.5}-\mathrm{RRL}_{\mathrm{pH} 7.0}\right)$ and $\mathrm{Al}$ stress $\left(\mathrm{RRL} 2,=\mathrm{RRL}_{\mathrm{pH} 4.5 \text { with Al }} \mathrm{Al}^{3+}-\mathrm{RRL}_{\mathrm{pH} 4.5 \text { without Al }} \mathrm{Al}^{3+}\right)$ were calculated using the average values from the five longest roots in each group. The root acid tolerance (RTI1) and Al tolerance (RTI2) indices were calculated as $\mathrm{RRL}_{\mathrm{pH} 4.5} / \mathrm{RRL}_{\mathrm{pH} 7.0}$ and $\mathrm{RRL}_{\mathrm{pH} 4.5+} 50$ $\mu \mathrm{MAl}^{3+} / \mathrm{RRL}_{\mathrm{pH} 4.5}$ (Taylor and Foy, 1985).

\section{Amplification and sequencing of the TaALMT1 gene promoters}

Genomic DNA was extracted from young seedlings using the $2 \mathrm{X}$ CTAB method, and the promoter sequences of the TaALMT1 gene were amplified by nested PCR using primer sets 1 and 2 (Sasaki et al., 2006). The primer sequences were as follows: 1F: 5'-gtcaccacgaagaagagg aacaccgacc-3'; 1R: 5'-agtggagcagcagcccgcagctggcgatg-3'; 2F: 5'-cctggttttettgatgggggcacacac-3'; and 2R: 5'-tgcccaccatctegecgtcgetctctct-3'.

All PCRs were performed in an ABI 9700 cycler (Applied Biosystems, USA) in a total volume of $25 \mu \mathrm{L}$ with 200 to $300 \mathrm{ng}$ total genomic DNA, $1.25 \mathrm{U}$ high-fidelity ExTaq polymerase (Takara, China), 1X ExTaq PCR buffer, $0.3 \mathrm{mM}$ each dNTP, $3 \mathrm{mM} \mathrm{MgCl}$, and $2 \mu \mathrm{M}$ each of primers $1 \mathrm{~F}$ and $1 \mathrm{R}$. The second PCR were performed using 10X dilutions of the first PCR reaction as template with $0.2 \mathrm{mM}$ dNTPs and $1.5 \mu \mathrm{M}$ each of primers $2 \mathrm{~F}$ and $2 \mathrm{R}$. The PCR conditions included an initial denaturation at $94^{\circ} \mathrm{C}$ for $2 \mathrm{~min}$; followed by 35 cycles at $94^{\circ} \mathrm{C}$ for $1 \mathrm{~min}$, $55^{\circ} \mathrm{C}$ (first round) or $57^{\circ} \mathrm{C}$ (second round) for $1 \mathrm{~min}$, and $72^{\circ} \mathrm{C}$ for $2 \mathrm{~min}$; and a final extension at $72^{\circ} \mathrm{C}$ for $5 \mathrm{~min}$. The PCR products were then separated on a $0.8 \%$ agarose gel and products of the right size were gel-extracted and ligated into the pMD18-T vector (TaKaRa). After transformation into Escherichia coli DH10B competent cells, three positive clones were randomly selected and sequenced from both directions. The sequence types were defined by comparison with the standard promoter sequence types I' (AB 243168), I (AB 243162), II (AB 243163), III (AB 243164), IV (AB 243165), V (AB 243166), and VI (AB 243167) (Sasaki et al., 2006).

\section{Multiple sequence alignment and phylogenetic analysis of $T a A L M T 1$ promoter sequences}

Sequence alignment of TaALMT1 promoters was performed using ClustalW (Thompson et al., 1994) and phylogenetic trees were constructed using MEGA 5.0 (Tamura et al., 2011). Sequences from 39 wheat cultivars, 53 landraces, and seven standard sequences were used to construct a topology tree to elucidate the evolutionary relationships among them, and the best models were tested. Models with the lowest Bayesian information criterion scores were considered to be the best substitution patterns. Maximum likelihood trees were constructed using the best models, and the complete deletion option was adopted for gaps in the aligned sequences. The evolutionary distance for each pair of aligned sequences was calculated from the best DNA model, and bootstrap values were estimated based on 1000 replications.

\section{Statistical analysis}

One-way analysis of variance was performed for RRL, RT1, and RTT2. The data for RRL1, RRL2, RTI1, and RTI2 were subjected to principal component analysis based on default variables in the NTSYSpc 2.1e software (Rohlf, 2000). The Al tolerance among wheat cultivars and landraces with different TaALMT1 promoter types were compared using the approximate $t$-test. 


\section{RESULTS}

\section{TaALMT1 promoter types in Sichuan-bred wheat cultivars and landraces}

Sasaki et al. (2006) designated seven TaALMT1 promoter types (I, I', II, III, IV, V, and VI) in wheat cultivars. Based on similarity when compared with standard promoter types, sequences of 92 Sichuan-bred wheat were classified into six types (I, I', II, III, IV, and V). These DNA sequences have been deposited into the GenBank database with accession numbers from JX977633 to JX977724.

Five promoter types were observed in 39 Sichuan-bred wheat cultivars, with type I' in 11, II in 14, III, and IV in 6 each, and V in 2 cultivars. However, in the landraces, only three promoter types were observed with I in 24, II in 26, and III in 3 landraces (Table 1).

Within each promoter type, about 0 to 6 bp substitutions/indels were observed (Table 1) and differences in the TaALMT1 promoters between cultivars and landraces were tested using the chi-square test (Table 2). These results showed that the promoter compositions between cultivars and landraces were significantly different $(\mathrm{P}>0.01)$ from each other.

Table 2. Composition of promoter sequences between Sichuan-bred wheat cultivars and landraces.
\begin{tabular}{lccccccc}
\hline Promoter sequence types & I & I' & II & III & IV & V & Total \\
\hline Cultivars & 0 & 11 & 14 & 6 & 6 & 2 & 39 \\
Landraces & 24 & 0 & 26 & 3 & 0 & 0 & 53 \\
Total & 24 & 11 & 40 & 9 & 6 & 2 & 92 \\
\hline
\end{tabular}

$\chi_{{ }_{(\text {Cultivar/Landrace) }}^{2}}^{2} 47.019^{* *}$, which indicates significance at $\mathrm{P}=0.01$ level.

\section{Comparison of Al tolerance among wheat lines with different $T a A L M T 1$ promoter types}

The Al tolerance (indicated by RTI2) among wheat lines with different TaALMT1 promoter types was compared (Table 3). As shown in Table 3, the Al tolerance index in landraces with promoter type I $(0.66)$ was significantly lower than in wheat lines with other promoter types. In addition, the Al tolerance in wheat cultivars with the type IV promoter $(0.85)$ was higher than in landraces with type II (0.77).

\begin{tabular}{|c|c|c|c|c|c|c|c|c|}
\hline \multicolumn{2}{|c|}{ Promoter sequence types } & \multirow{2}{*}{$\begin{array}{c}\text { Landraces } \\
\text { I } \\
0.66^{\mathrm{a}} / 24^{\mathrm{b}} \\
\end{array}$} & \multicolumn{6}{|c|}{ Cultivars } \\
\hline \multirow{2}{*}{ Landraces } & & & & & & & & \\
\hline & II $0.77 / 26$ & $* *$ & & & & & & \\
\hline \multirow{5}{*}{ Cultivars } & I' $0.76 / 11$ & $* *$ & $\begin{array}{l}\text { nis } \\
\text { ns }\end{array}$ & ns & & & & \\
\hline & II $0.78 / 14$ & $* *$ & ns & ns & ns & & & \\
\hline & III $0.75 / 6$ & * & ns & ns & ns & ns & & \\
\hline & IV $0.85 / 6$ & ** & * & ns & ns & ns & ns & \\
\hline & V $0.91 / 2$ & $* *$ & ns & ns & ns & ns & ns & ns \\
\hline
\end{tabular}

${ }_{\mathrm{a}, \mathrm{b}}$ Average of RTI2 and the number of clutivars/landraces with this type of promoter sequence. *, **, and ns indicate RTI2 values significant at $\mathrm{P}=0.05,0.01$, and not significant, respectively. 


\section{Comparison of root acid and Al tolerance between cultivars and landraces}

Root acid tolerance (indicated by RTI1) between cultivars and landraces was compared (Table 4). As shown in Table 4, the acid tolerance indices in cultivars (0.96) was significantly lower $(\mathrm{P}=0.01)$ than in landraces $(1.05)$, suggesting that landraces showed better acid tolerance than cultivars. No difference in acid tolerance was observed between the cultivars released by Sichuan (0.95) and Nationally (0.99). On the contrary, significant differences in acid tolerance $(\mathrm{P}=0.05)$ were observed among the landraces from different regions. Whereas the landraces from the south and west of Sichuan showed no difference in acid tolerance, both showed lower acid tolerance $(\mathrm{P}=0.05)$ than landraces from the east of Sichuan. Among those from the west, landraces from Ziyang (1.05) and Deyang (1.06) showed no difference in acid tolerance, but both showed significantly higher acid tolerance $(P=0.05)$ than landraces from Chengdu (1.03). Similarly, in the east, there was no difference in acid tolerance between the landraces from Nanchong (1.03) and Chongqin (1.02), and between landraces from Dazhou (1.20) and Bazhong (1.10). However, significant differences were observed between the landraces from Nanchong/Chongqin and Dazhou/Bazhong $(\mathrm{P}=0.01)$.

\begin{tabular}{|c|c|c|c|c|c|c|c|c|}
\hline Source & & & RTI1 & & & RTI2 & & \\
\hline Cultivars & & & $0.96^{\mathrm{Bb}}$ & & & $0.79^{\mathrm{Aa}}$ & & \\
\hline & By Sichuan Province (31) & & & $0.95^{\mathrm{Aa}}$ & & & $0.76^{\mathrm{Bb}}$ & \\
\hline \multirow{17}{*}{ Landraces } & By National Ministry of Agriculture (8) & & & $0.99^{\mathrm{Aa}}$ & & $0.86^{\mathrm{Aa}}$ & & \\
\hline & & & $1.05^{\mathrm{Aa}}$ & & & $0.73^{\mathrm{Ba}}$ & & \\
\hline & South Sichuan (23) & & & $1.04^{\mathrm{Ba}}$ & & & $0.76^{\mathrm{Aa}}$ & \\
\hline & & Yibin (10) & & & $1.01^{\mathrm{Aa}}$ & & & $0.77^{\mathrm{Aa}}$ \\
\hline & & Luzhou (7) & & & $1.05^{\mathrm{Aa}}$ & & & $0.76^{\mathrm{Aa}}$ \\
\hline & & Neijiang (5) & & & $1.06^{\mathrm{Aa}}$ & & & $0.72^{\mathrm{Aa}}$ \\
\hline & & Zigong (1) & & & $1.07--$ & & & $0.65--$ \\
\hline & West Sichuan (17) & & & $1.04^{\mathrm{Ba}}$ & & & $0.74^{\mathrm{Aa}}$ & \\
\hline & & Chengdu (10) & & & $1.03^{\mathrm{Bb}}$ & & & $0.75^{\mathrm{Aa}}$ \\
\hline & & Deyang (2) & & & $1.06^{\mathrm{Aa}}$ & & & $0.77^{\mathrm{Aa}}$ \\
\hline & & Ziyang (5) & & & $1.05^{\mathrm{Aa}}$ & & & $0.69^{\mathrm{Aa}}$ \\
\hline & East Sichuan (13) & & & $1.07^{\mathrm{Aa}}$ & & & $0.67^{\mathrm{Ba}}$ & \\
\hline & & Nanchong (5) & & & $1.03^{\mathrm{Bb}}$ & & & $0.67^{\mathrm{Aa}}$ \\
\hline & & Chongqin (2) & & & $1.02^{\mathrm{Bb}}$ & & & $0.67^{\mathrm{Aa}}$ \\
\hline & & Bazhong (2) & & & $1.10^{\mathrm{Aa}}$ & & & $0.66^{\mathrm{Aa}}$ \\
\hline & & Dazhou (3) & & & $1.20^{\mathrm{Aa}}$ & & & $0.60^{\mathrm{Bb}}$ \\
\hline & & Suining (1) & & & $0.99--$ & & & $0.98--$ \\
\hline
\end{tabular}

Significance of RTI values at $\mathrm{P}=0.05$ (upper case letters) and $\mathrm{P}=0.01$ level (lower case letters) are represented. (--) $=$ value acquired by only one cultivar/landrace and not used for multiple comparison.

Root Al tolerance (indicated by RTI2) between cultivars and landraces was also compared (Table 4$)$, and the cultivars $(0.79)$ showed better $\mathrm{Al}$ tolerance $(\mathrm{P}=0.05)$ than the landraces (0.73). Significant differences in Al tolerance were also observed between the cultivars released by Sichuan (0.76) and Nationally (0.86), which clearly shows that the cultivars released by Nationally had higher Al tolerance than the cultivars released by Sichuan. Among the landraces, significant differences in Al tolerance were observed. Whereas there was no difference in Al tolerance between the landraces from the south $(0.76)$ and the west $(0.74)$, both showed significantly better $\mathrm{Al}$ tolerance $(\mathrm{P}=0.05)$ than the landraces from the east $(0.67)$. Within the landraces from the south or west, there was no difference in $\mathrm{Al}$ tolerance. However, 
among those from the east, the landraces from Dazhou had the least Al tolerance when compared with the rest.

\section{Phylogenetic analysis of $T a A L M T 1$ gene promoter sequences}

The TaALMT1 gene promoter sequences from Sichuan-bred wheat cultivars and landraces were used for phylogenetic analysis. The best DNA models for cultivars, landraces, and combined cultivars and landraces all had T92 (Tamura 3-parameter), AICc values (Akaike Information Criterion, corrected) and maximum likelihood values ( $\mathrm{lnL}$ ) of $5626.58 /-2728.14,5423.80 /-2600.70$, and 7775.07/-3691.19, respectively.

The phylogenetic tree based on TaALMT1 promoter sequences from 53 landraces formed two separate clades (Figure 1A), with promoter type III in one branch supported by a high bootstrap value, and landraces with promoter types I and II clustering in another branch. Within the clade formed by types I and II, the landraces were randomly distributed.

The phylogenetic tree based on TaALMT1 promoter sequences from 39 cultivars formed two separate branches (Figure 1B). Cultivars with type III formed a separate clade, whereas cultivars with promoter types I', II, IV, and V clustered together. Within the clade formed by types I', II, IV, and V, cultivars with promoter types IV and V formed a subclade, and cultivars with types I' and II formed a second subclade.

The phylogenetic tree using TaALMT1 gene promoter sequences from the 92 Sichuan-bred wheat lines, including all cultivars and landraces, formed two separate clades (Figure 1C). Interestingly, three landraces and six cultivars with promoter type III clustered together into a separate clade. In addition, wheat lines with promoter types I', I, II, IV, and V clustered together, forming a parallel clade. Within this branch, type I' formed an individual inner branch with a high bootstrap value and stemmed from the branch formed by types I and II, whereas types IV and V formed a separate branch with low bootstrap values.

\section{Principal component (PC) analysis of tolerance data}

An acidic $\mathrm{pH}$ condition is a prerequisite for $\mathrm{Al}$ toxicity. To understand the association between acid tolerance and $\mathrm{Al}$ tolerance, principal component analysis was performed using the root re-growth lengths at acid $\mathrm{pH} 4.50$ (RRL1), and acid $\mathrm{pH} 4.50$ with $50 \mu \mathrm{M} \mathrm{Al}^{3+}$ (RRL2), and tolerance indices of acid (RTI1) and Al (RTI2). The results showed that variations in tolerance levels were caused by the first $(55.49 \%)$ and second (36.91\%) principal components and totaled to $92.40 \%$ of the variation (Table 5). The first PCs (PC1) had positive eigenvectors for RRL1 and RTI1 and negative for RRL2 and RTI2, and were considered mainly as Al tolerance components. The second PCs (PC2), causing $36.91 \%$ of the variation and all positive eigenvector values, were mainly elucidated as components conferring tolerance to low $\mathrm{pH}$. Based on the pattern and values of $\mathrm{PC} 1$ and $\mathrm{PC} 2, \mathrm{Al}$-tolerant wheat was mainly distributed in region a with promoter types I', II, III, IV, and V, whereas the Al-sensitive wheat was mainly distributed in region $\mathrm{b}$ with promoter types I and II. The intermediate wheat was located at region $\mathrm{c}$ and the majority in region d were with promoter types I' and II (Figure 2). However, the acid-tolerant wheat (underlined) was mainly distributed in region d with promoter types I and II (Figure 2). 
(A)

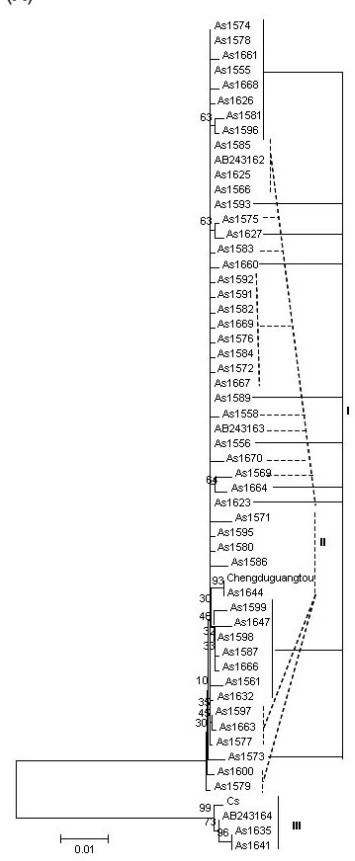

(B)

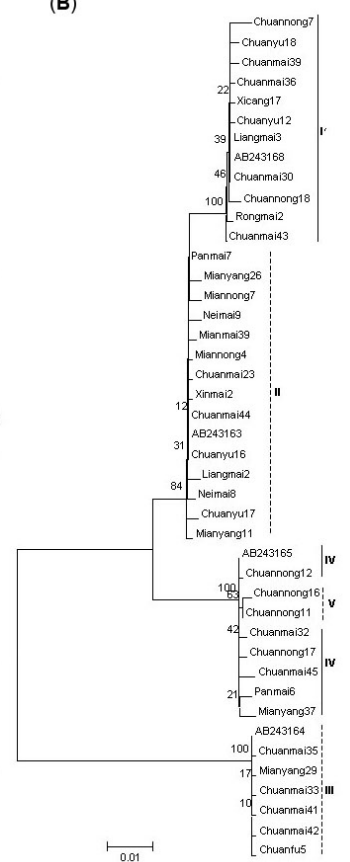

(C)

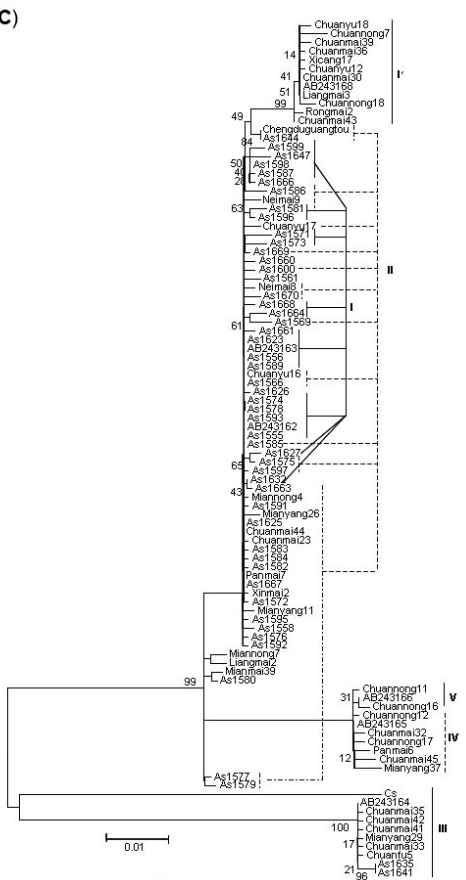

Figure 1. Phylogenetic analysis based on the promoter sequences of TaALMT1 genes from 39 cultivars (A), 53 landraces (B), and combined cultivars and landraces (C). 
Table 5. Eigenvalues and eigenvectors in principal coordination analysis.

\begin{tabular}{lcccc}
\hline Principal coordination & PC1 & PC2 & PC3 & PC4 \\
\hline Eigenvalue & 2.22 & 1.48 & 0.28 & 0.028 \\
Percentage & $55.49 \%$ & $36.91 \%$ & $9.89 \%$ & $10 \%$ \\
Cumulative & $55.49 \%$ & $92.40 \%$ & $99.29 \%$ & \\
Eigenvector & & & 0.34 & 0.02 \\
RRL1 & 0.35 & 0.87 & -0.13 & -0.02 \\
RRL2 & -0.90 & 0.41 & -0.38 & -0.12 \\
RTI1 & 0.69 & 0.62 & -0.03 & \\
RTI2 & -0.90 & 0.41 & & \\
\hline
\end{tabular}

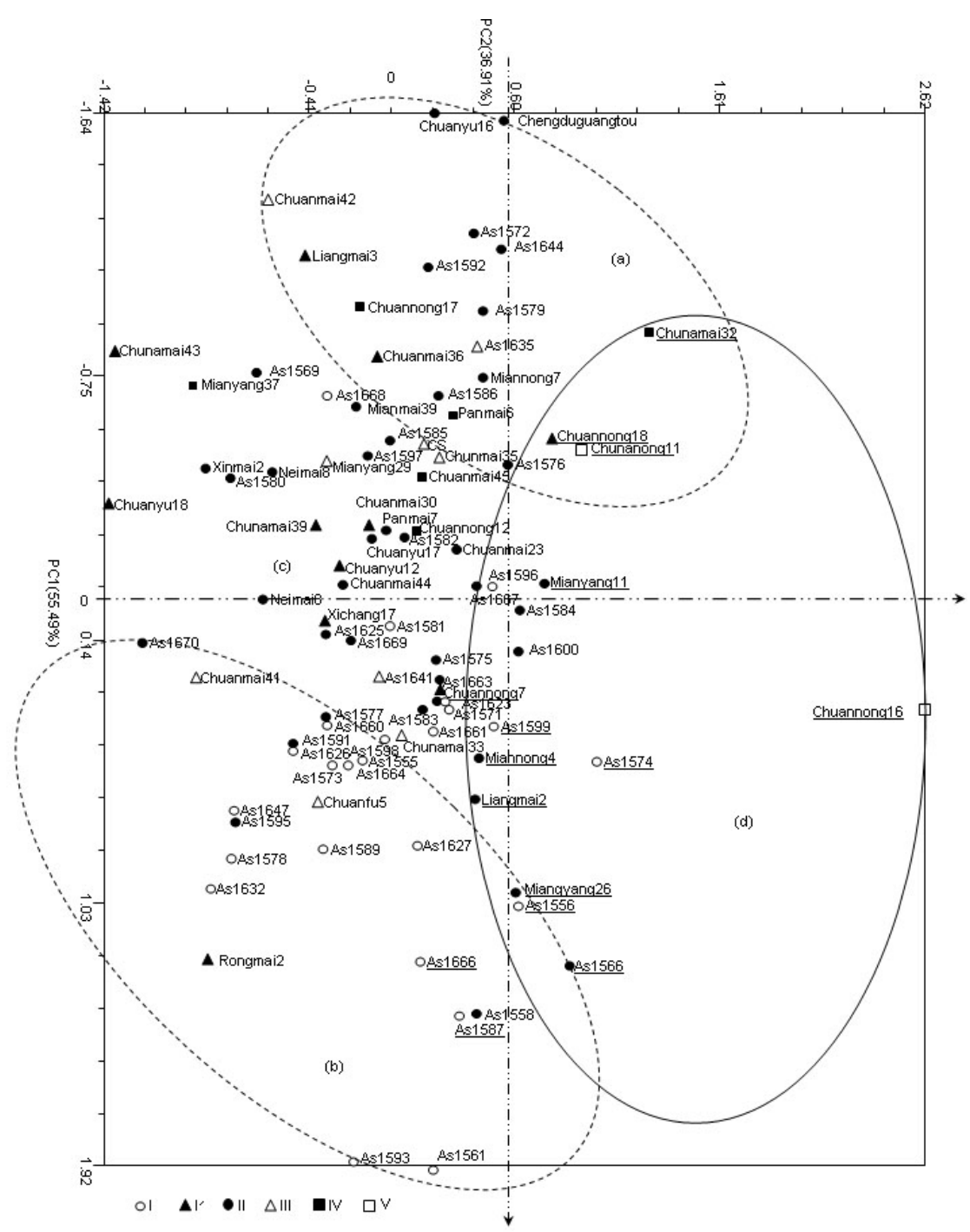

Figure 2. Scores for principal components (PC) 1 and 2. 


\section{DISCUSSION}

Al toxicity is a major limiting factor for root growth in acidic soils, and it is wise to plant Al-tolerant wheat in these soils to decrease the loss in wheat yield. Therefore, the identification of new Al-tolerant varieties is important to provide genetic diversity in wheat germplasms. Genetic analysis of various wheat genotypes collected worldwide revealed that $\mathrm{Al}^{3+}$ tolerance might have originated independently in the USA, Brazil, and China (Hu et al., 2008; Raman et al., 2008). However, to date, only the Al-tolerant wheat lines in Brazil have been studied extensively (Berzonsky, 1992; Riede and Anderson, 1996; De Sousa, 1998; Ma et al., 2005). Thus far, only limited numbers of wheat lines from China have been evaluated for $\mathrm{Al}$ tolerance (Hu et al., 2008), although significant genetic variation in $\mathrm{Al}$ tolerance has occurred among wheat landraces and cultivars (Stodart et al., 2007; Zhou et al., 2007). Here, we investigated the $\mathrm{Al}$ tolerance and acid tolerance of Sichuan-bred wheat cultivars and landraces from China. Compared with the Al-tolerant control CS (RRL2 $\geq-1.18$ and RTI2 $\geq 0.84$ ), some Sichuan-bred wheat lines (mainly in region a of Figure 2), such as Chengduguangtou, As1644, As1572, As1635, As1579, As1592, As1586, As1576, Chuanyu16, Chuanmai32, Chuannong16, Chuanmai42, Chuannong11, Liangmai3, Miannong7, Chuannong18, Chuannong17, Chuanmai36, and Panmai6 (the only one outside of region a), were identified to have high or moderate levels of Al tolerance. Besides this, some acid-tolerant wheat lines, such as As1556, As1566, As1574, As1587, As1599, As1666, Chuanmai 32, Chuanmai 33, Chuannong 11, Chuannong 16, Chuannong 18, Chuannong 7, Liangmai 2, Miannong 4, Mianyang 11, and Mianyang 26 (RRL1 $\geq 0$ and RTI2 $\geq 1$, mainly in region d of Figure 2), were also identified.

The composition and variation in TaALMT1 gene promoter sequences from 92 Sichuan-bred wheat cultivars and landraces were also investigated. Interestingly, the cultivars had five promoter types (I', II, III, IV, and V), whereas the landraces had three promoter types (I, II, and III). None of the Sichuan-bred wheat landraces or cultivars had promoter type VI. Within each promoter type, 0 to $6 \mathrm{bp}$ differences were observed in the sequences. In a previous study, lines with promoter type I showed the lowest levels of TaALMT1 expression and Al tolerance, whereas lines with types V and VI (three replicates) showed the highest expressions levels and Al tolerance. Accordingly, lines with types III and IV (two replicates) showed intermediate levels of TaALMT1 expression and Al tolerance (Sasaki et al., 2006). In our study, Sichuan-bred wheat landraces with promoter type I showed significantly lower Al tolerance than the landraces or cultivars with other promoter types (Table 3).

Currently, lines with TaALMT1 promoter allele III, with intermediate levels of $T a$ $A L M T$ expression and Al tolerance, have been reported only in the wheat landrace CS endemic to Sichuan, China (Yen et al., 1988; Sasaki et al., 2006). In this study, two additional landraces (As1635 and As1641) and six cultivars (Chuanmai 35, Chuanmai 33, Chuanmai 41, Chuanmai 42, Chuanfu 5, and Mianyang 29) with promoter type III were identified, suggesting that Sichuan-bred wheat landraces are one of the sources for promoter type III. In addition, promoter type III in Sichuan-bred wheat cultivars could have likely originated from Sichuan-bred wheat landraces. The six cultivars with promoter type III were produced by the breeding Institute of Chengdu, and Mianyang of Sichuan in China. The two wheat landraces CS and As1635 carrying promoter type III were found close to these breeding institutes. However, pedigree analysis could not confirm that the promoter type III in these cultivars was derived from CS 
or As1635. Because there is no report yet on the existence of promoter type III in wheat lines collected outside of China, we presume that the most likely sources for promoter type III in Sichuan-bred cultivars are the landraces like CS and As1635.

We have shown that the landraces have better acid tolerance than the cultivars. On the contrary, the cultivars exhibited better Al tolerance than the landraces (Table 4). In addition, between cultivars released by Sichuan and Nationally, there was no difference in acid tolerance, but significant differences in $\mathrm{Al}$ tolerance were observed. Within landraces, those from East Sichuan showed better acid tolerance but less Al tolerance than landraces from South and West Sichuan.

\section{ACKNOWLEDGMENTS}

Research supported by National Key Technologies R\&D Program (\#2011BAD35B03), Education Department of Sichuan Province (\#09ZA077 and \#09ZZ024).

\section{REFERENCES}

Berzonsky WA (1992). The genomic inheritance of aluminum tolerance in "Atlas 66" wheat. Genome 35: 689-693.

Chen Q, Sun Y and Dong Y (1985). Cytogenetic studies on interspecific hybrids of Xinjiang wheat. Acta Agron. Sin. 11: 23-28.

Dai SF, Yan ZH, Liu DC, Zhang LQ, et al. (2009). Evaluation on Chinese bread wheat landraces for low pH and aluminum tolerance using hydroponic screening. Agric. Sci. China 8: 285-292.

De Sousa CNA (1998). Classification of Brazilian wheat cultivars for aluminium toxicity in acid soils. Plant Breed. 117: 217-221.

Delhaize E, Ryan PR, Hebb DM, Yamamoto Y, et al. (2004). Engineering high-level aluminum tolerance in barley with the ALMT1 gene. Proc. Natl. Acad. Sci. U. S. A. 101: 15249-15254.

Dong YS, Zheng DS, Qiao DY, Zeng XQ, et al. (1981). Investigation and study on Yunnan wheat (Triticum aestivum ssp. yunnanense King). Acta Agron. Sin. 7: 145-151.

Garvin DF and Carver BF (2003). Role of the Genotypes in Tolerant to Acidity and Aluminum Toxicity. In: Handbook of Soil Acidity (Rengel Z, ed.). Marcel Dekker, New York, 387-406.

Hu SW, Bai GH, Carver BF and Zhang DD (2008). Diverse origins of aluminum-resistance sources in wheat. Theor. Appl. Genet. 118: 29-41.

Kochian LV (1995). Cellular mechanisms of aluminum toxicity and resistance in plants. Annu. Rev. Plant Physiol. Plant Mol. Biol. 46: 237-260.

Luo MC and Dvorák J (1996). Molecular mapping of an aluminum tolerance locus on chromosome 4D of Chinese Spring wheat. Euphytica 91: 31-35.

Ma HX, Bai GH, Carver BF and Zhou LL (2005). Molecular mapping of a quantitative trait locus for aluminum tolerance in wheat cultivar Atlas 66. Theor. Appl. Genet. 112: 51-57.

Mclay CDA, Ritchie GSP, Porte WM and Cruse A (1994). Amelioration of subsurface acidity in sandy soils in low rainfall regions II. Changes to soil solution composition following the surface application of gypsum and lime. Aust. J. Soil Res. 32: 847-865.

Milla R and Gustafson JP (2001). Genetic and physical characterization of chromosome 4DL in wheat. Genome 44: 883892.

Raman H, Zhang K, Cakir M, Appels R, et al. (2005). Molecular characterization and mapping of ALMT1, the aluminiumtolerance gene of bread wheat (Triticum aestivum L.). Genome 48: 781-791.

Raman H, Ryan PR, Raman R, Stodart BJ, et al. (2008). Analysis of TaALMT1 traces the transmission of aluminum resistance in cultivated common wheat (Triticum aestivum L.). Theor. Appl. Genet. 116: 343-354.

Riede CR and Anderson JA (1996). Linkage of RFLP markers to an aluminum tolerance gene in wheat. Crop Sci. 36: 905-909.

Rohlf FJ (2000). NTSYS-pc: Numerical Taxonomy and Multivariate Analysis System. Version 2.1. Exeter Software: Setauket, New York. 
Sasaki T, Yamamoto Y, Ezaki B, Katsuhara M, et al. (2004). A wheat gene encoding an aluminum-activated malate transporter. Plant J. 37: 645-653.

Sasaki T, Ryan PR, Delhaize E, Hebb DM, et al. (2006). Sequence upstream of the wheat (Triticum aestivum L.) ALMT1 gene and its relationship to aluminum resistance. Plant Cell Physiol. 47: 1343-1354.

Shao QQ, Li CS and Basang CR (1980). Semi-wild wheat from Xizang (Tibet). Acta Genet. Sin. 7: 149-156.

Stodart BJ, Raman H, Coombes N and Mackay M (2007). Evaluating landraces of bread wheat Triticum aestivum L. for tolerance to aluminium under low pH conditions. Genet. Resour. Crop Evol. 54: 759-766.

Tamura K, Peterson D, Peterson N, Stecher G, et al. (2011). MEGA5: molecular evolutionary genetics analysis using maximum likelihood, evolutionary distance, and maximum parsimony methods. Mol. Biol. Evol. 28: 2731-2739.

Taylor GJ and Foy CD (1985). Mechanisms of aluminum tolerance in Triticum aestivum L. (wheat). I. Differential pH induced by winter cultivars in nutrient solutions. Am. J. Bot. 72: 695-701.

Thompson JD, Higgins DG and Gibson TJ (1994). CLUSTAL W: improving the sensitivity of progressive multiple sequence alignment through sequence weighting, position-specific gap penalties and weight matrix choice. Nucleic Acids Res. 22: 4673-4680.

von Uexküll HR and Mutert E (1995). Global extent, development and economic impact of acid soils. Plant Soil 171: 1-15.

Yamaguchi M, Sasaki T, Sivaguru M, Yamamoto Y, et al. (2005). Evidence for the plasma membrane localization of Alactivated malate transporter (ALMT1). Plant Cell Physiol. 46: 812-816.

Yen C, Luo MC and Yang JL (1988). The Origin of the Tibetan Weedrace of Hexaploid Wheat, Chinese Spring, Chengduguangtou and other Landraces of White Wheat Complex from China. Proceedings of the 7th. International Wheat Genetics Symposium (Miller TE and Koebner RMD, eds.). Institute of Plant Sciences Research, Cambridge, 175-179.

Zhou LL, Bai GH, Carver B and Zhang DD (2007). Identification of new sources of aluminum resistance in wheat. Plant Soil 297: 105-118. 